\title{
SKIN Detection of Animation ChaRACTERS
}

\author{
Kazi Tanvir Ahmed Siddiqui ${ }^{1}$ and Abu Wasif ${ }^{2}$ \\ ${ }^{1}$ Department of Electrical and Computer Engineering, North South University, Dhaka, \\ Bangladesh \\ ${ }^{2}$ Department of Computer Science and Engineering, Bangladesh University of \\ Engineering and Technology, Dhaka, Bangladesh
}

\begin{abstract}
The increasing popularity of animes makes it vulnerable to unwanted usages like copyright violations and pornography. That's why, we need to develop a method to detect and recognize animation characters. Skin detection is one of the most important steps in this way. Though there are some methods to detect human skin color, but those methods do not work properly for anime characters. Anime skin varies greatly from human skin in color, texture, tone and in different kinds of lighting. They also vary greatly among themselves. Moreover, many other things (for example leather, shirt, hair etc.), which are not skin, can have color similar to skin. In this paper, we have proposed three methods that can identify an anime character's skin more successfully as compared with Kovac, Swift, Saleh and Osman methods, which are primarily designed for human skin detection. Our methods are based on RGB values and their comparative relations.
\end{abstract}

\section{KEYWORDS}

Animation; Skin color detection; Image Processing; Skin color classifier; RGB based skin detection

\section{INTRODUCTION}

Skin identification from an image is the precondition of various applications such as face detection and recognition, obscene video recognition, people tracking, hand gesture recognition and mood identification. Face detection/recognition is used in almost everywhere, for search engines, digital cameras, and surveillance systems. But, these techniques are made for humans only. Most of the cases, these methods do not work on animes, cartoons or on comic characters.

Characters in cartoons, comics and games are collectively called cartoon characters [1]. Currently, Google image search can detect human faces but are unable to detect animation faces [2]. Many different techniques are used for skin detection modeling [3-8]. Simpler of those models are based on several threshold values [5-8], while more complex models use Neural Networks [9], Bayesian classifier [10], max entropy [11], k-means clustering [12].

Skin color varies greatly not only for humans but also for animation characters. So, it is very challenging to set a rigorous condition in which skins of all animation characters fit. Skin color differs greatly in different illumination conditions. Another challenge is that, many objects have also skin like color, e.g. wood, leather, hair, sand, skin colored clothing etc. [8]. Even animation characters have different skin colors based on race, gender, and age. 
In this paper, we have proposed three simple methods based on RGB range and their comparative relations. We have used means, standard deviations and ranges from the skin of the cartoon characters to specify the conditions. We have compared our results with other human-skin detection methods [5-8] and an animation-skin detection method [1] and presented the results.

The database we have used is also compiled by us. We have used the Google search engine, and an online database [13] to accumulate the images used in our experiment. We have also annotated skins manually using Adobe Photoshop to set the accurate skin pixels.

\section{RELATED WORKS}

\subsection{Human Skin Detection Methods}

There have been several methods for human skin detection. Some popular methods are Kovac et al. [5], Saleh [6], Swift [7], Osman et al. [8], Omanovic et al. [14] etc. These all methods are based on specific RGB range, RGB ratios, and their comparative relations. These methods are described below:

According to Kovac method, a pixel is skin if:

- $\mathrm{R}>95$ and $\mathrm{G}>40$ and $\mathrm{B}>20$ and

- $\operatorname{Max}(\mathrm{R}, \mathrm{G}, \mathrm{B})-\operatorname{Min}(\mathrm{R}, \mathrm{G}, \mathrm{B})>15$ and

- $|\mathrm{R}-\mathrm{G}|>15$ and

- $\quad \mathrm{R}>\mathrm{G}$ and $\mathrm{R}>\mathrm{B}$

According to Swift method, a pixel is not skin pixel if:

- $\mathrm{B}>\mathrm{R}$ or

- $\mathrm{G}<\mathrm{B}$ or

- $\mathrm{G}>\mathrm{R}$ or

- $\mathrm{B}<\mathrm{R} / 4$ or

- $\mathrm{B}>200$

According to Saleh method, a pixel is skin if:

- $\quad \mathrm{R}-\mathrm{G}>20$ and

- $\mathrm{R}-\mathrm{G}<80$

Osman et al. [8] and Omanovic et al. [14] methods are based on RGB ratio.

According to Osman method, a pixel is skin if:

- $0.0 \leq(\mathrm{R}-\mathrm{G}) /(\mathrm{R}+\mathrm{G}) \leq 0.5$ and

- $\mathrm{B} /(\mathrm{R}+\mathrm{G}) \leq 0.5$

Omanovic et al. [14] tested with various RGB ratios and showed their performance accordingly. 


\subsection{Cartoon character skin detection method}

Anime-skins are not identical to human skins. They are drawn by artists and their color varies greatly based on age, race, gender, illumination condition etc. Takayama et al. [1] presented a way of detecting anime-skin. Their method uses HSV values.

According to Takayama et al. [1], a pixel is skin if:

- Hue is between $\mathbf{0}$ to $\mathbf{4 0}$ degrees and

- Value is more than $\mathbf{7 5 \%}$

This method uses Canny method [15] for edge extraction, and then, the skin region segmentation is done by the flood-fill approach.

\section{MethodologY}

The purpose of this paper is to establish a method for skin extraction from animation or cartoon images. The total task can be divided into four steps:

1. Data collection

2. Data preparation

3. RGB based skin detection methods

4. Testing and Evaluation

\subsection{Data Collection}

There are no suitable databases for animation-images. So, we created our own database, which consists of 255 jpeg. images of anime characters. The database is compiled by images collected from an anime database [13] and google image search [2]. Some properties of our database are:

- All the images are color images, no grayscale or binary images were used.

- Images are of both genders.

- Images are of different illumination conditions e.g. images were taken at morning, dusk and night.

- Image characters are from different races e.g. White, Black, and Asian.

- Backgrounds of the images are cluttered and also of uniform color.

- Some images consist of multiple characters while others single characters.

- Characters in the images have varied hair color: black, white, brown, gray, red etc.

- Some subjects of the images have spectacles, headbands, armbands and tattoos.

Image size ranged from dimensions $104 \times 99$ to $1003 \times 1200$. We have used 70 images from the 255 images to set the conditions for our methods.

\subsection{Data Preparation}

An accurate image segmentation is one of the most important steps to establish the ground truth information and to get the most accurate result [4]. In order to annotate skin from image, human intervention is required. We have annotated 70 images manually using Adobe Photoshop CS6. We have used the magic wand tool of that software to manually select skin areas. It is very difficult, even for humans, to identify whether some pixels are skin or not, because of the 
different illumination conditions and shadows. Moreover, our images are of anime characters. These images were drawn by humans. Hence, we have established some rules to maintain the integrity while annotating images:

- Edges were not considered as skin.

- Shaded skin were considered as skin.

- Where cloth or hair meet skin, those pixels were considered skin based on the difference between other skin pixels. If the difference were too much then they were not counted as skin.

We have worked on $\mathbf{7 0}$ images, a total of $\mathbf{1 8 , 0 0 0 , 5 9 3}$ pixels. We colored those skin pixels using RGB values of: $(\mathbf{0 , 2 5 5 , \boldsymbol { 0 } )}$ or $(\mathbf{2 5 5}, \mathbf{2 5 5}, \mathbf{0})$. These specific values were used so that, the correct amount and location of each skin pixel can be obtained. Next, we compared that specific pixel with our method's found skin pixel to ensure that we have identified correctly or not.

We used MATLAB R2014a software to ensure whether our manually annotated skin pixels are detected as skin or not. We observed some anomalies (i.e. some manually colored skin pixels were not detected). That is why, considering some variations, to find all of the skin pixels, we have fixed the range of RGB values according to Table 1 .

We used two colors: green $(0,255,0)$ and yellow $(255,255,0)$ to paint the skins, so that while extracting, skin color does not combine with other background colors.

Table 1. Algorithm for extracting skin pixels from annotated images
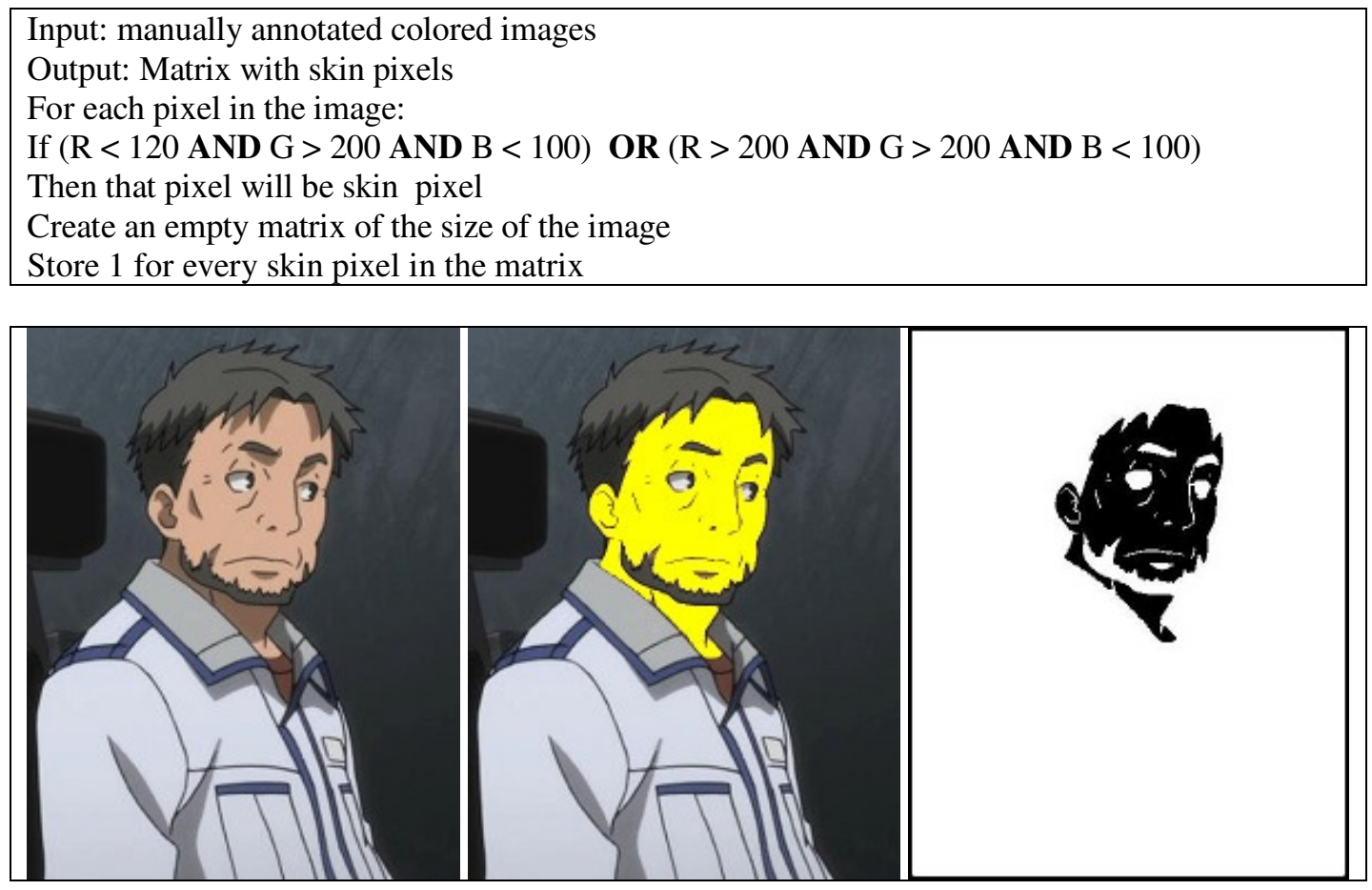

Figure 1. Actual image

Figure 2. Skin colored manually Figure 3. Manually colored image's skin extracted using Algorithm of Table 1. 
Figure 1 shows the actual image before coloring, Figure 2 shows that image colored using Adobe Photoshop (only the skin portion) and Figure 3 shows how the algorithm of Table 1 detects the ground truth of skin pixels from the colored image.

We checked all the images manually to ensure that, every image's skin has been identified correctly.

\subsection{RGB based skin detection algorithm}

In designing our algorithm, we have taken into consideration that skin color varies greatly according to race, gender and illumination conditions. We have collected RGB values from the skin pixels of different images. And then, we used the range, mean and deviations of those values to specify the conditions for detecting skin.

Based on the range and mean of the RGB values of skin and their comparative relations, we have specified some conditions to be skin. One version of the algorithm is described in Table 2

Table 2. Proposed Method-I for Detecting Skin Pixels

Input: Animation images

Output: 2D matrix where 1 is for skin, 0 is for non-skin

For each pixel in the image:

If $(120<\mathrm{R}<255$ AND $90<\mathrm{G}<250$ AND $70<\mathrm{B}<218$ AND $\mathrm{R}>\mathrm{G}+10$ AND $\mathrm{G}>\mathrm{B}+10)$

Then that pixel is skin

Create an empty matrix equals the size of the image

Store 1 for every skin pixel

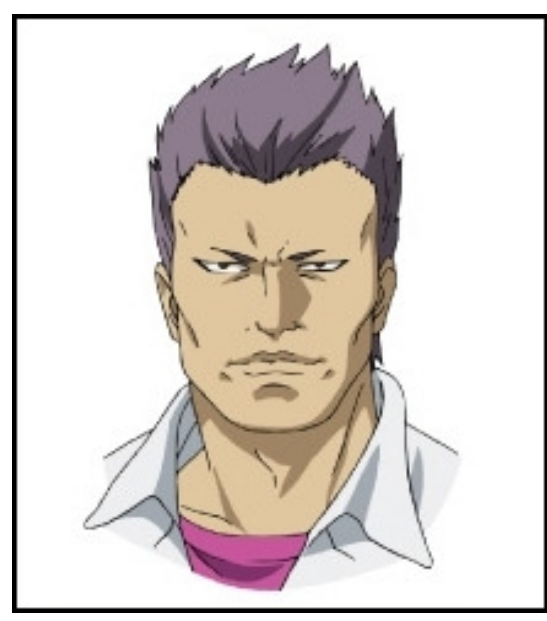

Figure 4. Actual image

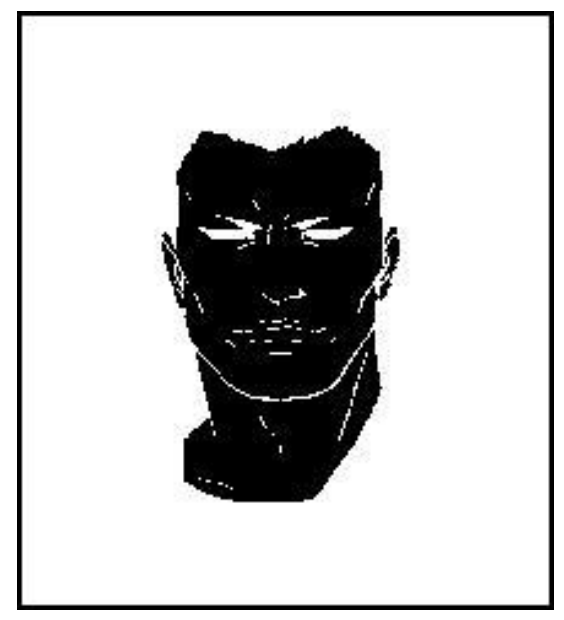

Figure 5. Skin detected using Table 2 
International Journal on Soft Computing (IJSC) Vol.6, No. 1, February 2015

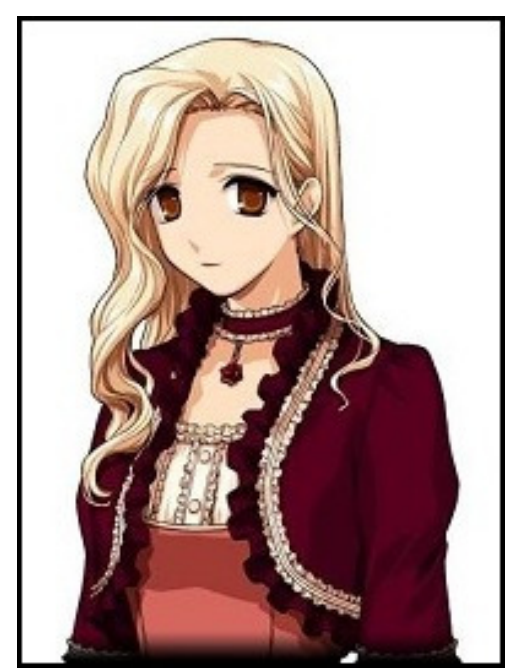

Figure 6. Actual image

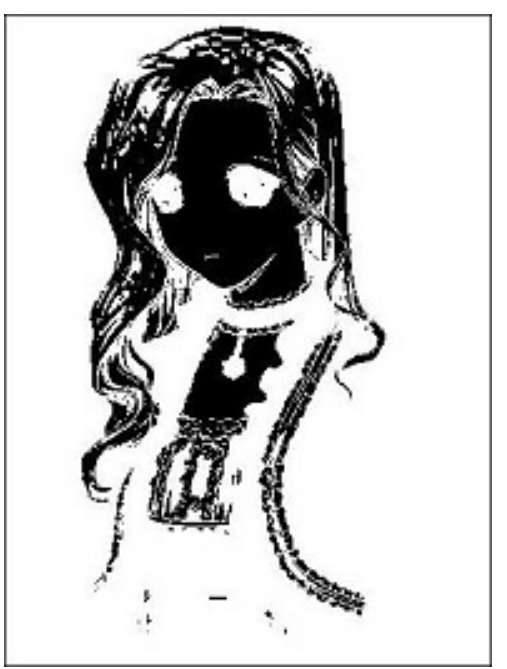

Figure 7. Skin detected using Table 2

We see that in Figure 5 almost all the skin pixels have been detected. But, in Figure 7, in addition to skin pixels, some hair and dress pixels also have been detected as skin. This is due to the fact that, hair color of this subject is similar to its skin color.

We have changed the conditions slightly to increase the true positive. The changed condition is described in Table 3 and Table 4. These new conditions increase our TP from $79.54 \%$ to $83.91 \%$ (Table 3) and $88.3 \%$ (Table 4) but also increased the FP from $9.5 \%$ to $10.98 \%$ (Table 3) and $14.2 \%$ (Table 4).

Table 3. Proposed Method-II for Detecting Skin Pixels

Input: Animation images

Output: 2D matrix where 1 is for skin, 0 is for non-skin

For each pixel in the image:

If $(120<\mathrm{R}<255$ AND $90<\mathrm{G}<250$ AND $70<\mathrm{B}<218$ AND $\mathrm{R}>\mathrm{G}+10$ AND $\mathrm{G}>\mathrm{B})$

Then that pixel is skin

Create an empty matrix that equals the size of the image

Store 1 for every skin pixel

Table 4. Proposed Method-III for Detecting Skin Pixels

Input: Animation images

Output: 2D matrix where 1 is for skin and 0 is for non-skin

For each pixel in the image:

If $(120<\mathrm{R}<255$ AND $90<\mathrm{G}<250$ AND $70<\mathrm{B}<218$ AND $\mathrm{R}>\mathrm{G}$ AND $\mathrm{G}>\mathrm{B})$

Then that pixel is skin

Create an empty matrix equals the size of the image

Store 1 for every skin pixel 


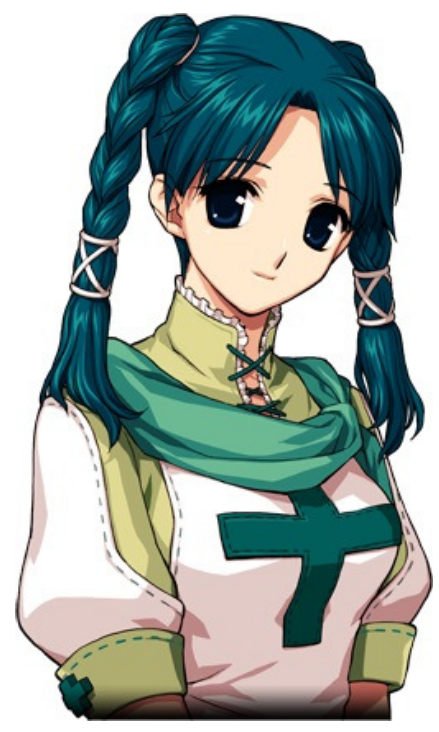

Figure 8. Actual image

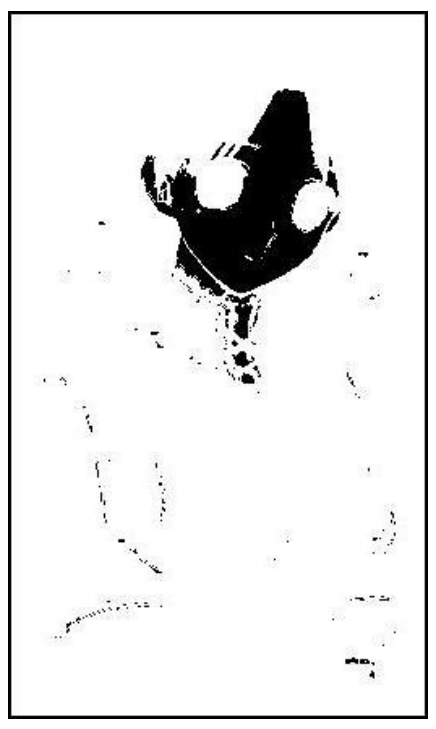

Figure 9. Skin detected using Table 2

Figure 8 shows the actual image. Figure 9, Figure 10 and Figure 11 show distinct differences (shown in red circles). These figures show that though there is a marked increase in TP; FP is also increased. Figure 12, 13, 14 and 15 show that while Method-I has been failed to detect some of the skin pixels, Method-II and Method III have been successful.

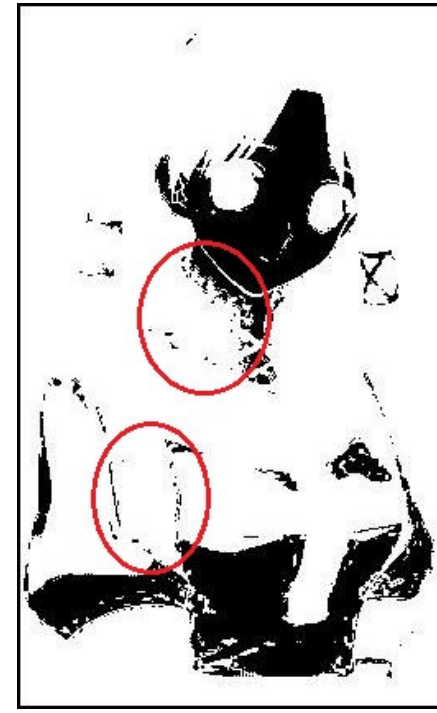

Figure 10. Skin detected using Table 3

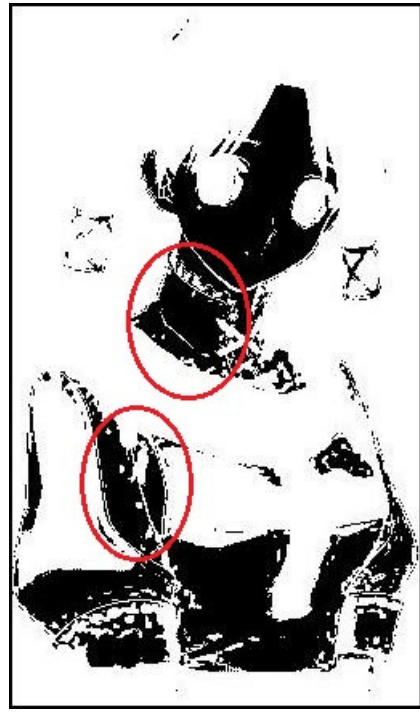

Figure 11. Skin detected using Table 4 


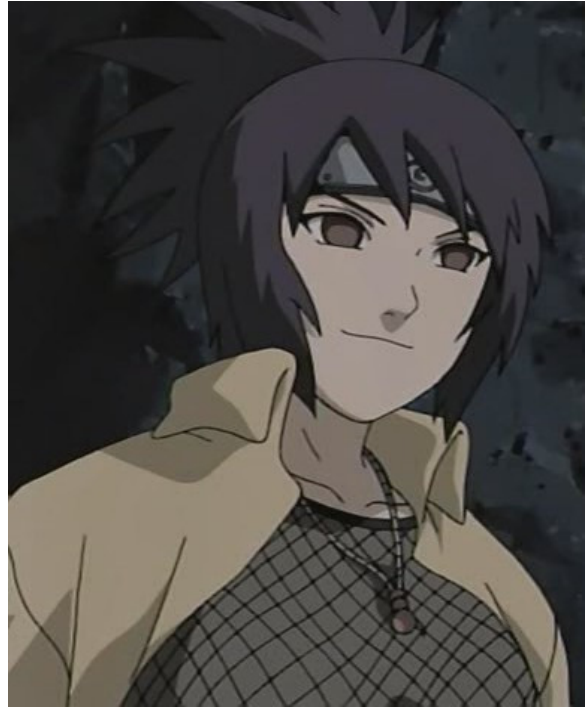

Figure 12. Original image

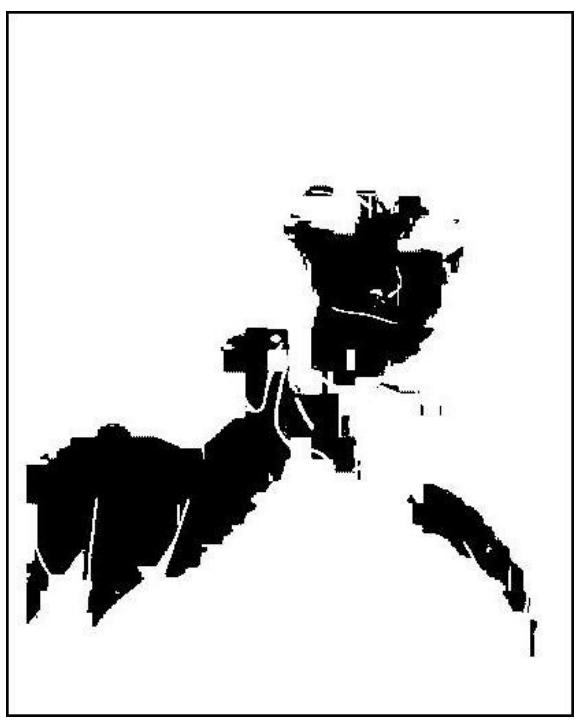

Figure 14. Skin detected using Table 3

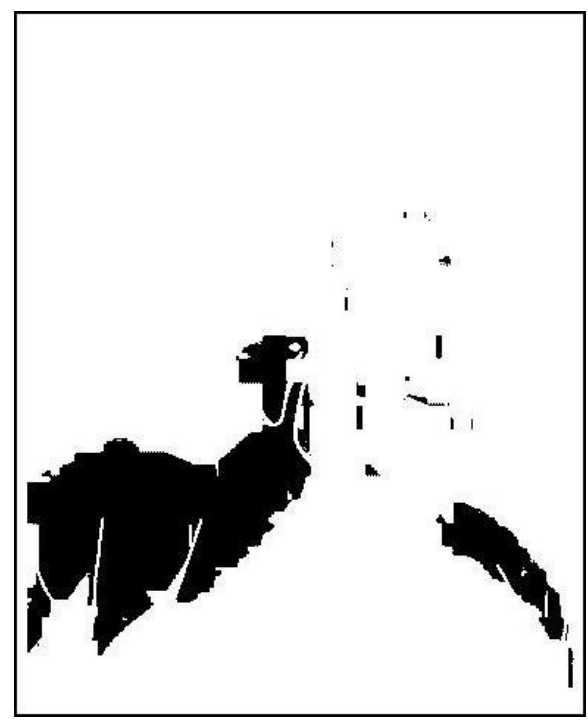

Figure 13. Skin detected using Table 2

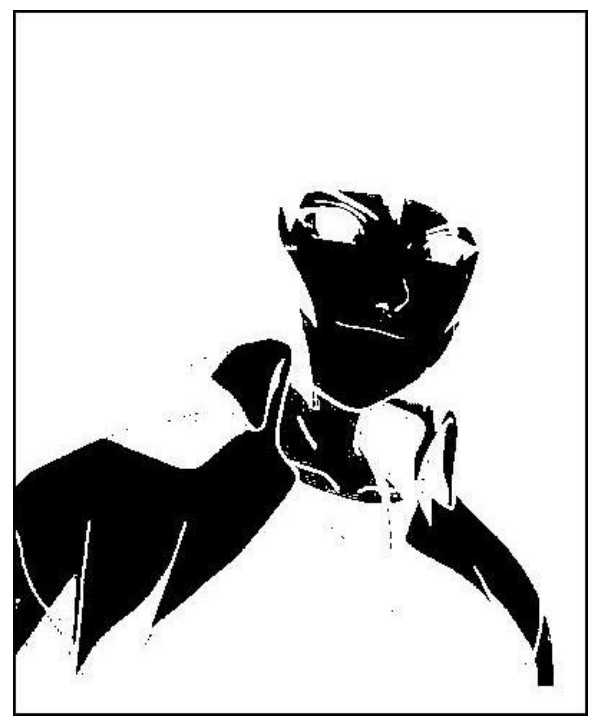

Figure 15. Skin detected using Table 4

We have also used Kovac et al. [5], Saleh [6], Osman et al. [8], and Takayama et al. [1] classifiers to detect skin and compared their performances accordingly.

\subsection{Testing and Evaluation}

Quantitative and qualitative methods are widely used to measure the performance of skin color detection algorithms [16]. The qualitative technique is concerned with the human observation or visual inspection based evaluation of the classifier.

The rate of True and False Positives are used as the quantitative measures. We have used both quantitative and qualitative methods to evaluate our algorithm's performance. After evaluating the algorithm as described in Table 2, two new variants (as described in Table 3 and Table 4) are proposed. 
For performance measurement, True Positive (TP) and False Positive (FP) are defined, in the context of our experiment, as:

$$
\begin{gathered}
\mathrm{TP}=\frac{\text { number of pixels detected as skin }}{\text { total number of skin pixels }} \\
\mathrm{FP}=\frac{\text { number of non-skin pixels detected as skin }}{\text { total number of pixels in an image }}
\end{gathered}
$$

Table 5. Method to find TP and FP

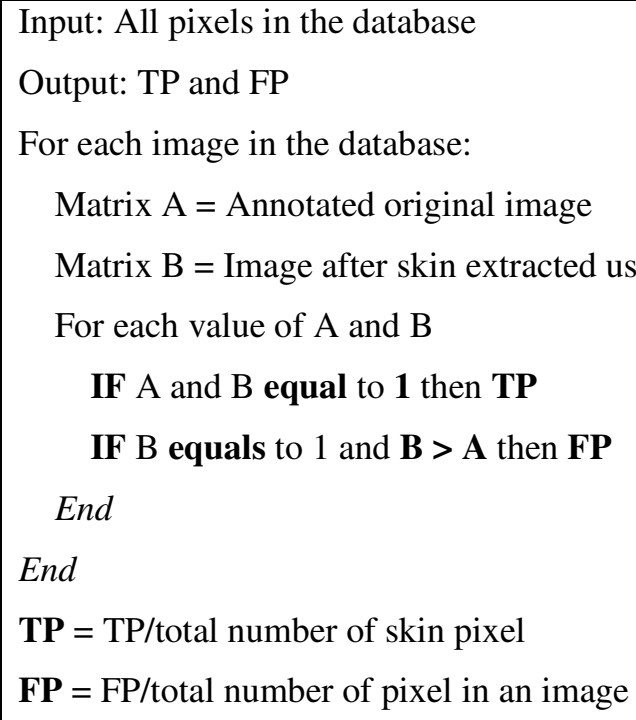

Table 5 is used to find the TP and FP from the whole database by comparing every image after finding skin with manually annotated image. We have used three methods (Table 1, 2 and 3), alongside Osman et al. [1], Kovac et al. [5], Saleh [6], Swift [7], and Omanovic et al. [14]. We have shown TP and FP of all methods.

\section{RESULT AND DISCUSSION}

The performance of different methods of skin color detection, i.e. the three variants of our proposed technique, as well as Kovac, Saleh, Osman and Takayama methods are shown in Table 6 . We have used all these methods on the same datasets and presented the results accordingly. Fig. 16 to Fig. 19 show some examples of qualitative measurement. Here skin pixels are marked as black, and non-skin pixels are white. These examples used for qualitative measurements support what is shown by the comparative analysis of the quantitative measurements found experimentally.

A good skin color detection algorithm should have high rates of true positives and low rates of false positives. In general, there is a trade-off between the two rates. From the experimental results, as presented in Table 6, it is evident that among the three proposed methods, Method-III has the highest True Positive (TP), which is good. However, it also has bigger false positives (FP) compared to Method-I and Method-II. On the other hand, Method-I has the smallest FP, but it has 
lower TP compared to Method-II and Method-III. Method-I outperforms the Takayama and the Saleh method in terms of TP, but is outperformed by the Kovac and the Osman method.

Method-III outperforms all the methods, except the Osman method, in terms of TP. However, Method-III is superior to the Osman method in terms of FP, because Method-III's FP is much smaller as compared to the Osman method, which means although the Osman method correctly detects more skin pixels than Method-III, it will also make more mistakes in falsely detecting non-skin pixels as skin pixels. Overall, Method-III is a competent skin detection algorithm, and all the three proposed algorithms are reasonable performers on the basis of experimental results compared to the existing algorithms.

Table 6. Performance of Skin Color Classifiers

\begin{tabular}{|l|l|l|l|l|}
\hline Methods & True Positive & Percent(TP) & $\begin{array}{l}\text { False } \\
\text { Positive }\end{array}$ & Percent(FP) \\
\hline Method-I & 1389666 & $79.54 \%$ & 1711682 & $9.5 \%$ \\
\hline Kovac & 1504259 & $86.1 \%$ & 2644507 & $14.7 \%$ \\
\hline Osman & 1716094 & $98.23 \%$ & 7794816 & $43.30 \%$ \\
\hline Takayama & 1325582 & $75.88 \%$ & 1392439 & $7.7 \%$ \\
\hline Saleh & 1369588 & $78.40 \%$ & 2521295 & $14.01 \%$ \\
\hline Method-II & 1465885 & $83.91 \%$ & 1976905 & $10.98 \%$ \\
\hline Method-III & 1542610 & $88.3 \%$ & 2570836 & $14.2 \%$ \\
\hline
\end{tabular}

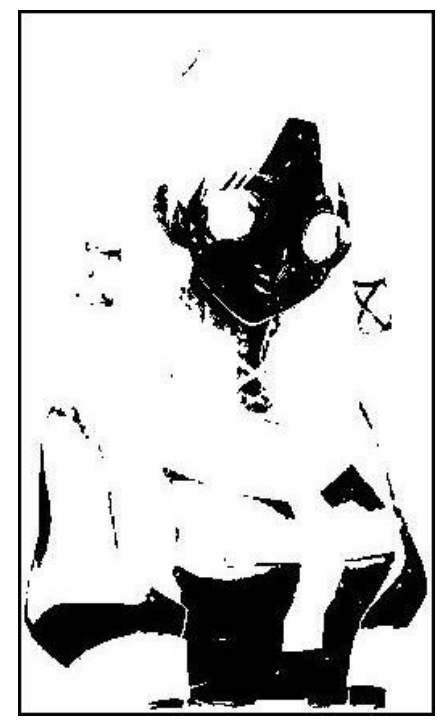

Figure 16. Skin detected using Kovac

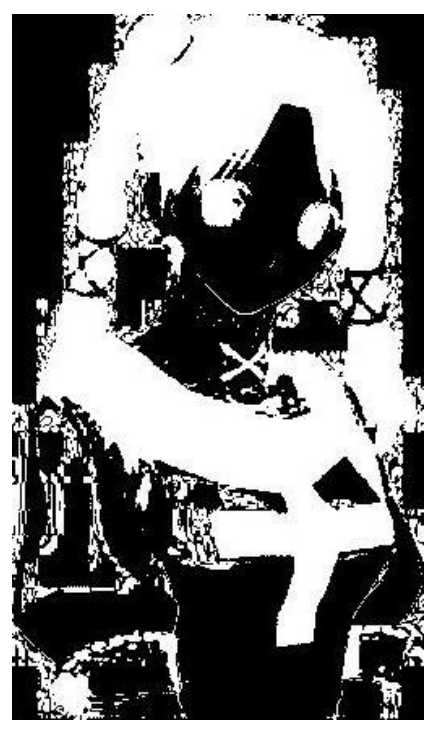

Figure 17. Skin detected using Osman 
International Journal on Soft Computing (IJSC) Vol.6, No. 1, February 2015

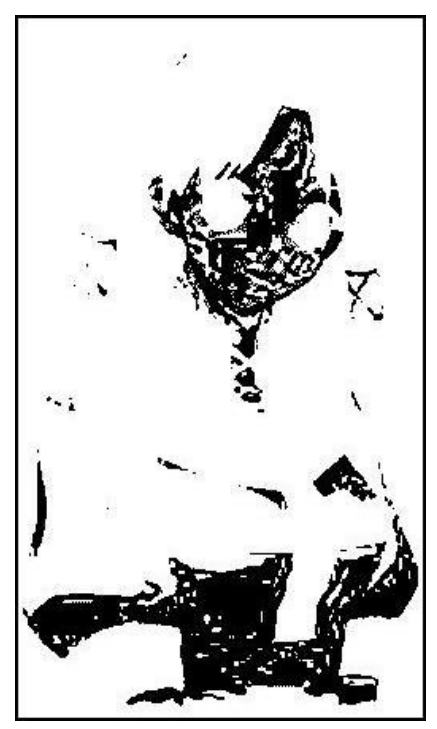

Figure 18. Skin detected using Saleh

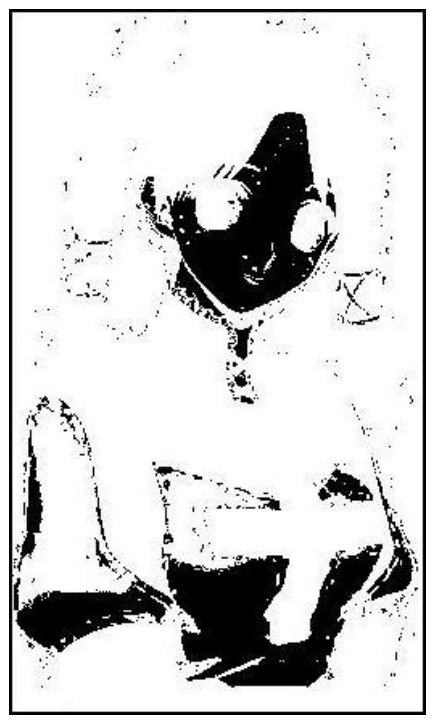

Figure 19. Skin detected using Takayama

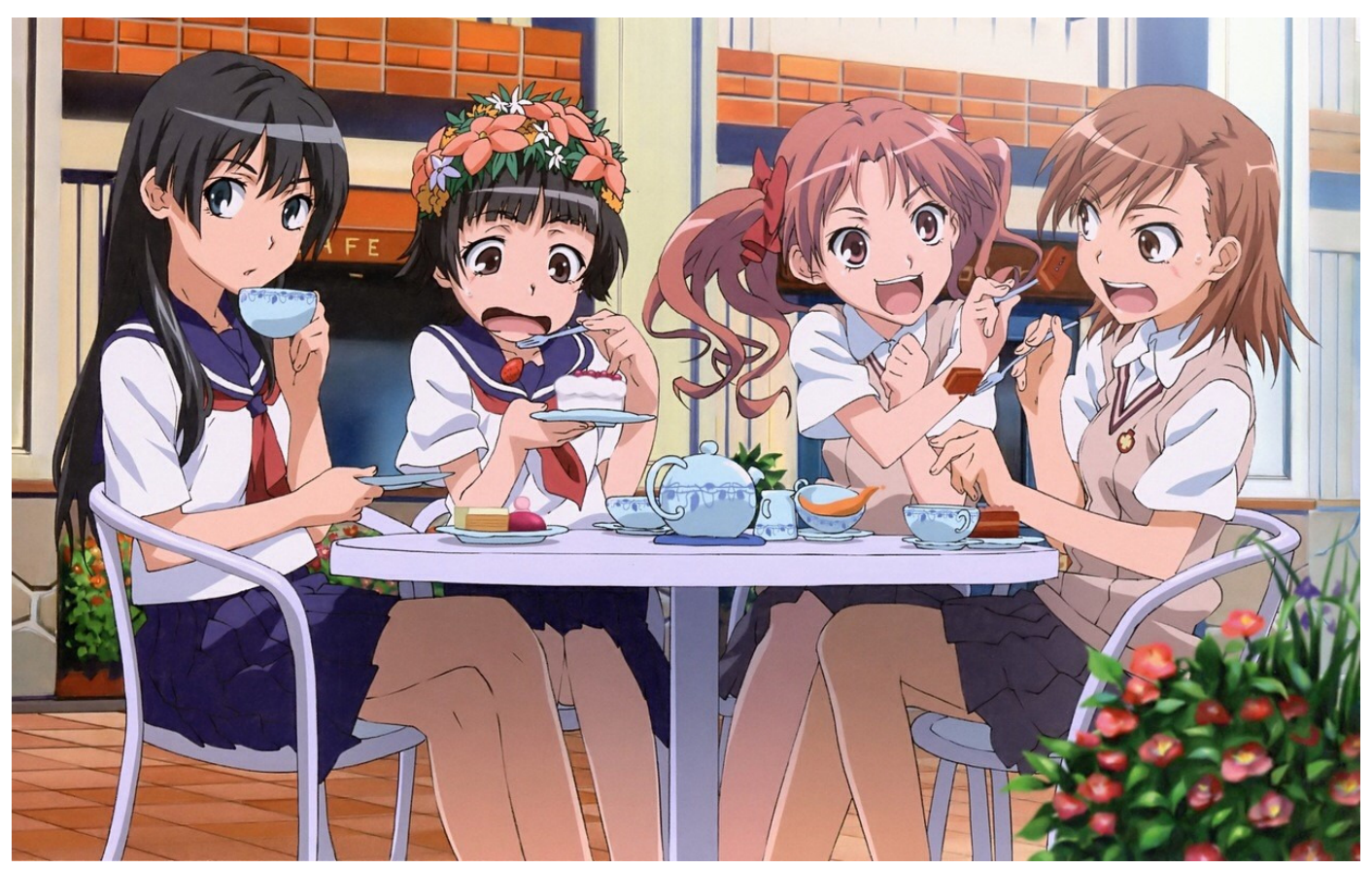

Figure 20. Original Image (multiple characters) 
International Journal on Soft Computing (IJSC) Vol.6, No. 1, February 2015

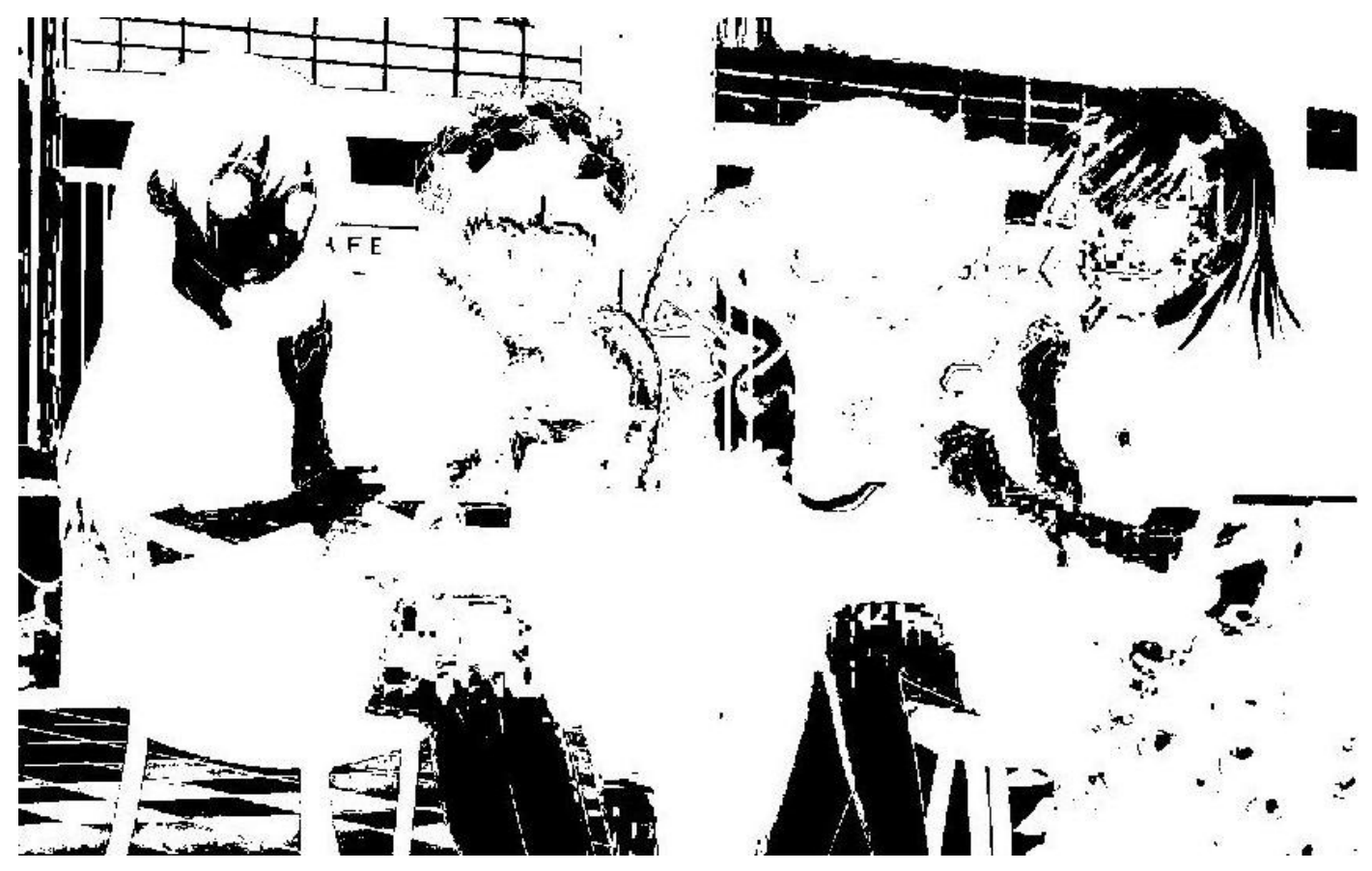

Figure 21. Skin extracted using Method-I

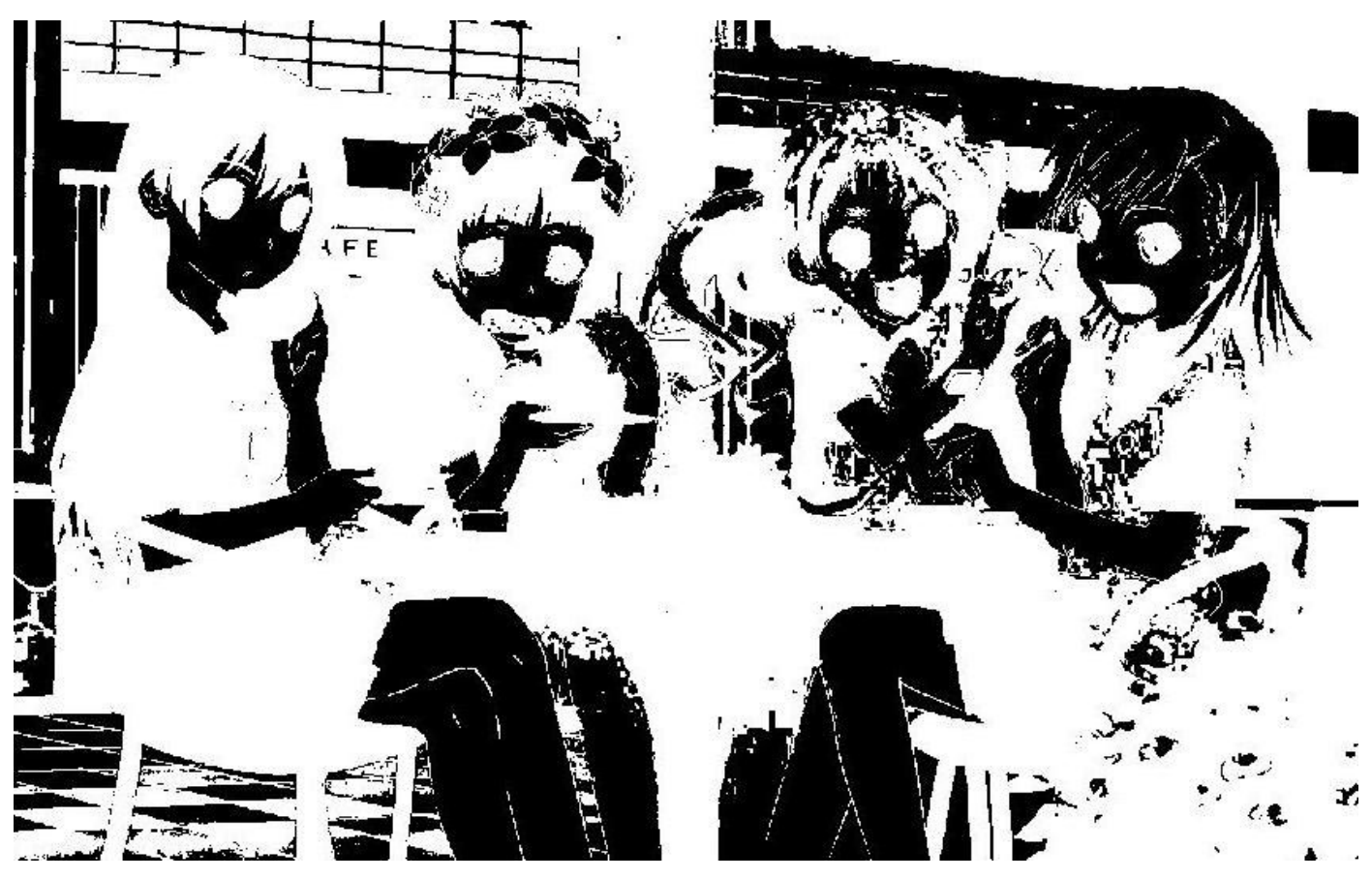

Figure 22. Skin extracted using Method-II 
International Journal on Soft Computing (IJSC) Vol.6, No. 1, February 2015

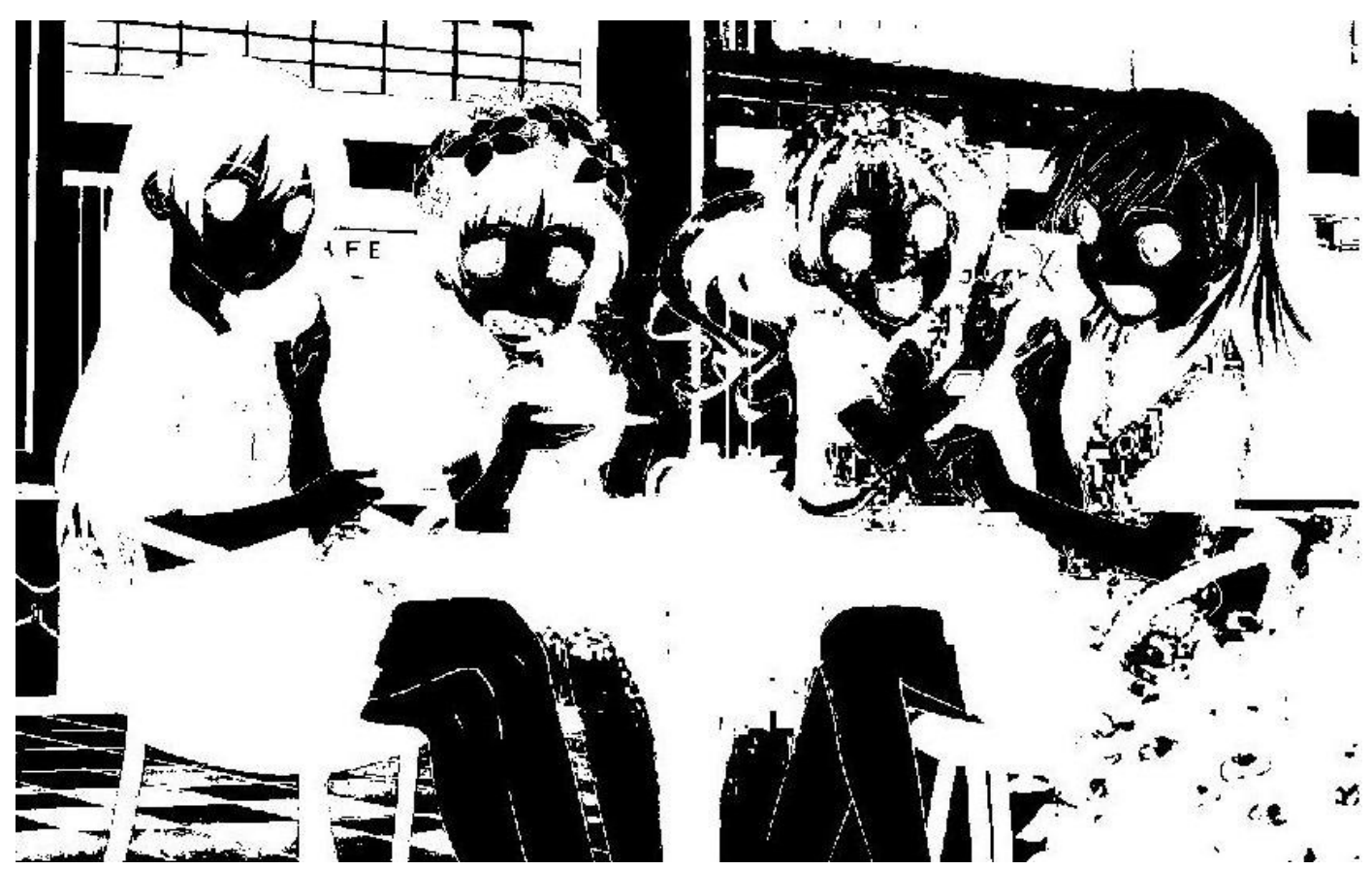

Figure 23. Skin extracted using Method-III

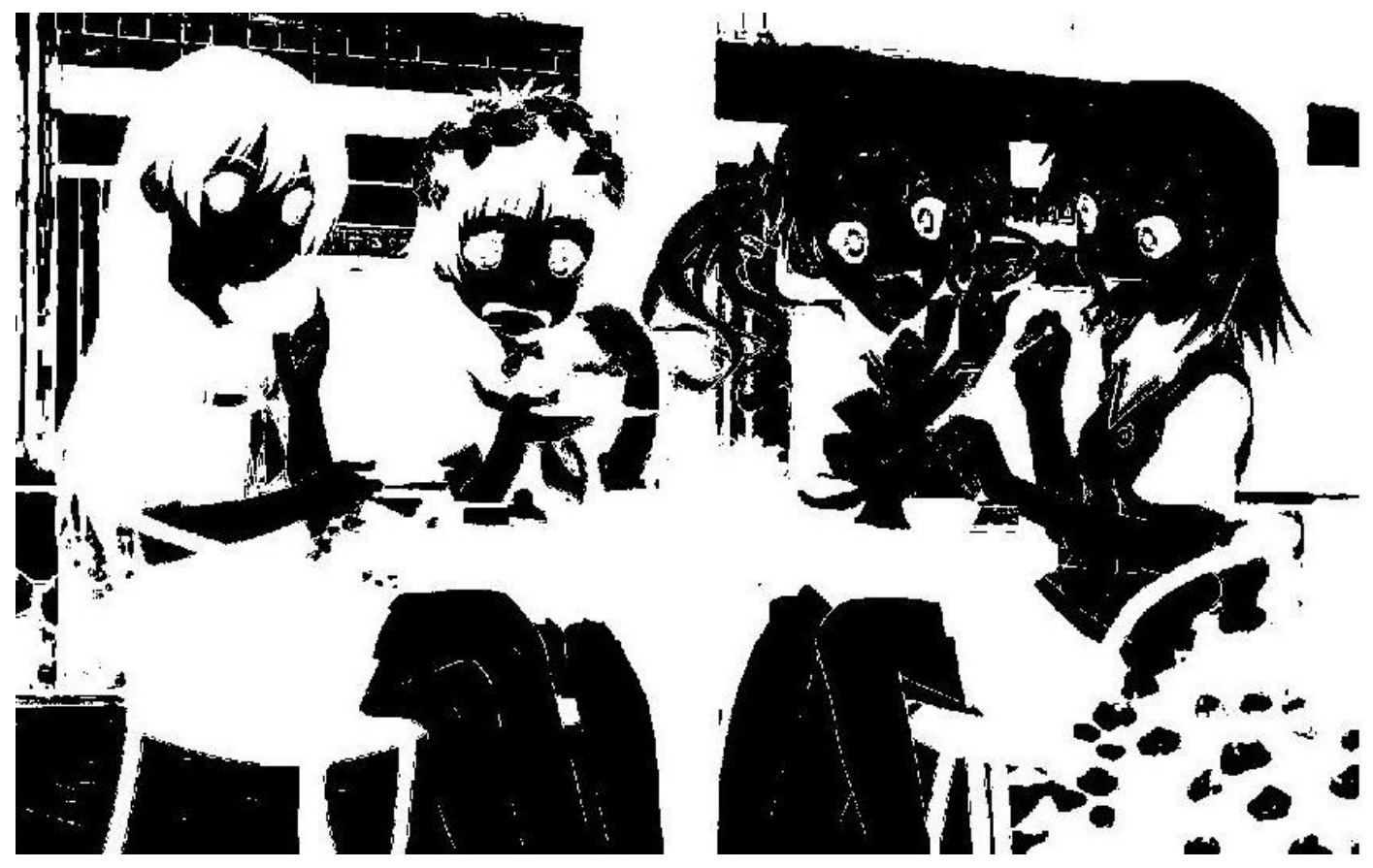

Figure 24. Skin extracted using Kovac method 


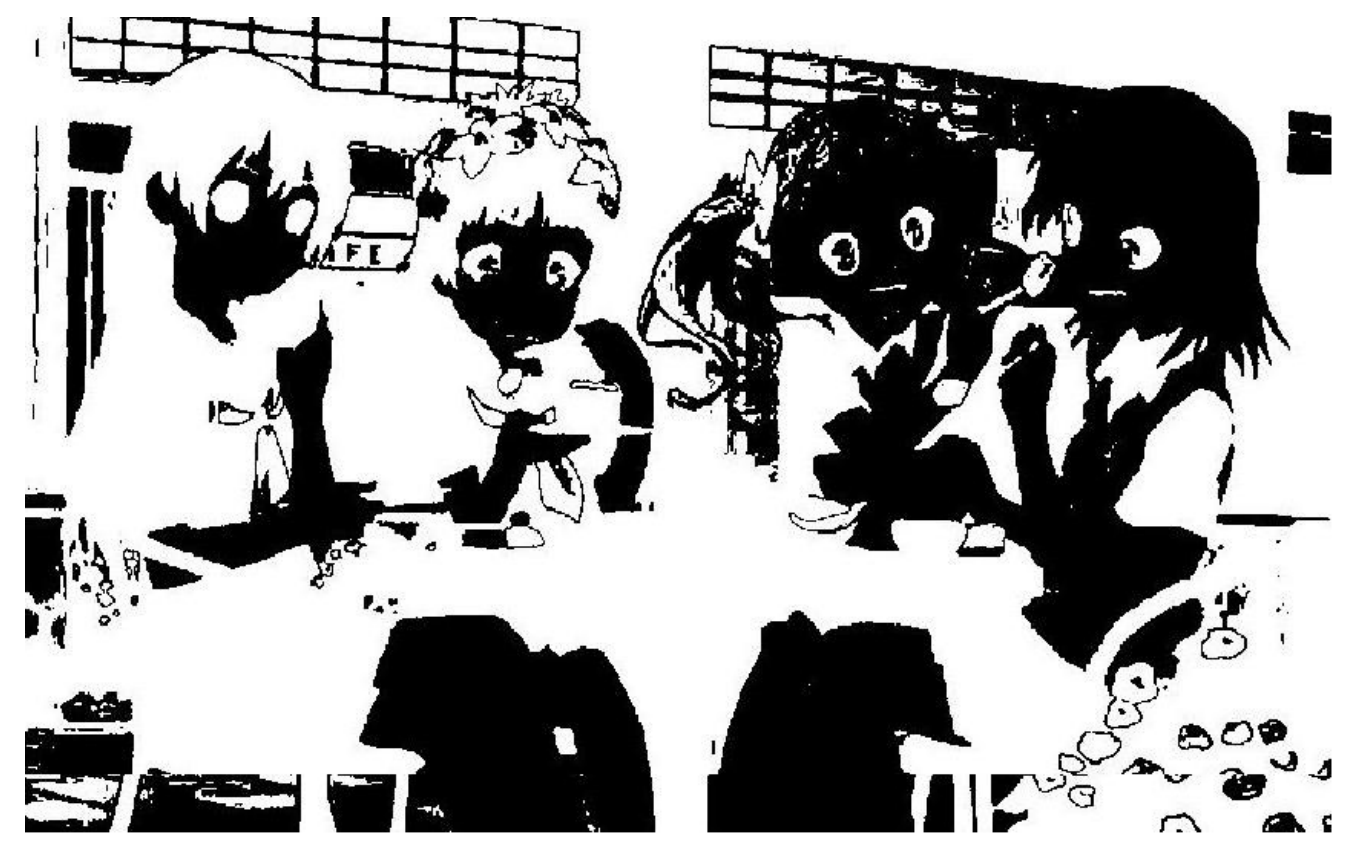

Figure 25. Skin extracted using Osman method

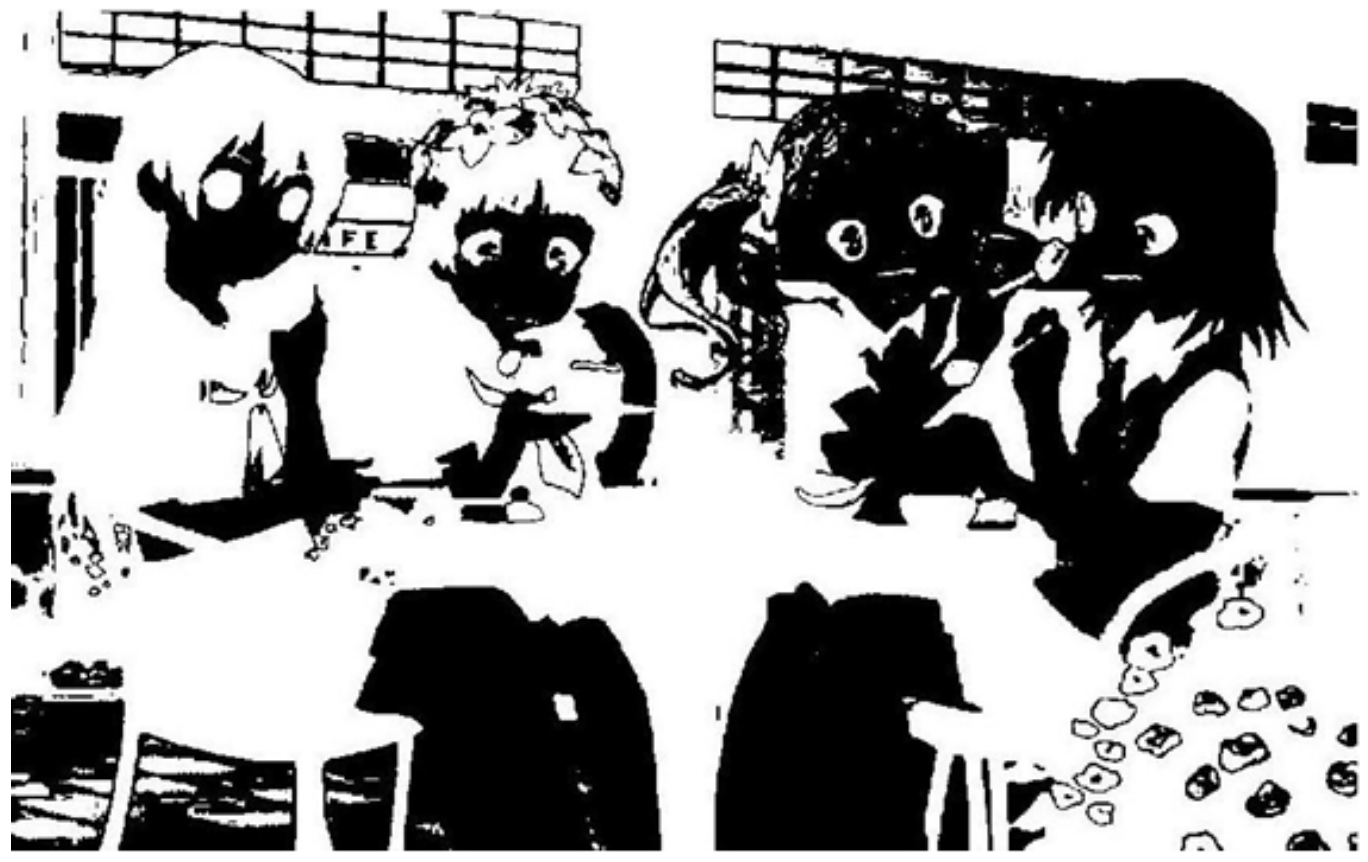

Figure 26. Skin extracted using Saleh method 


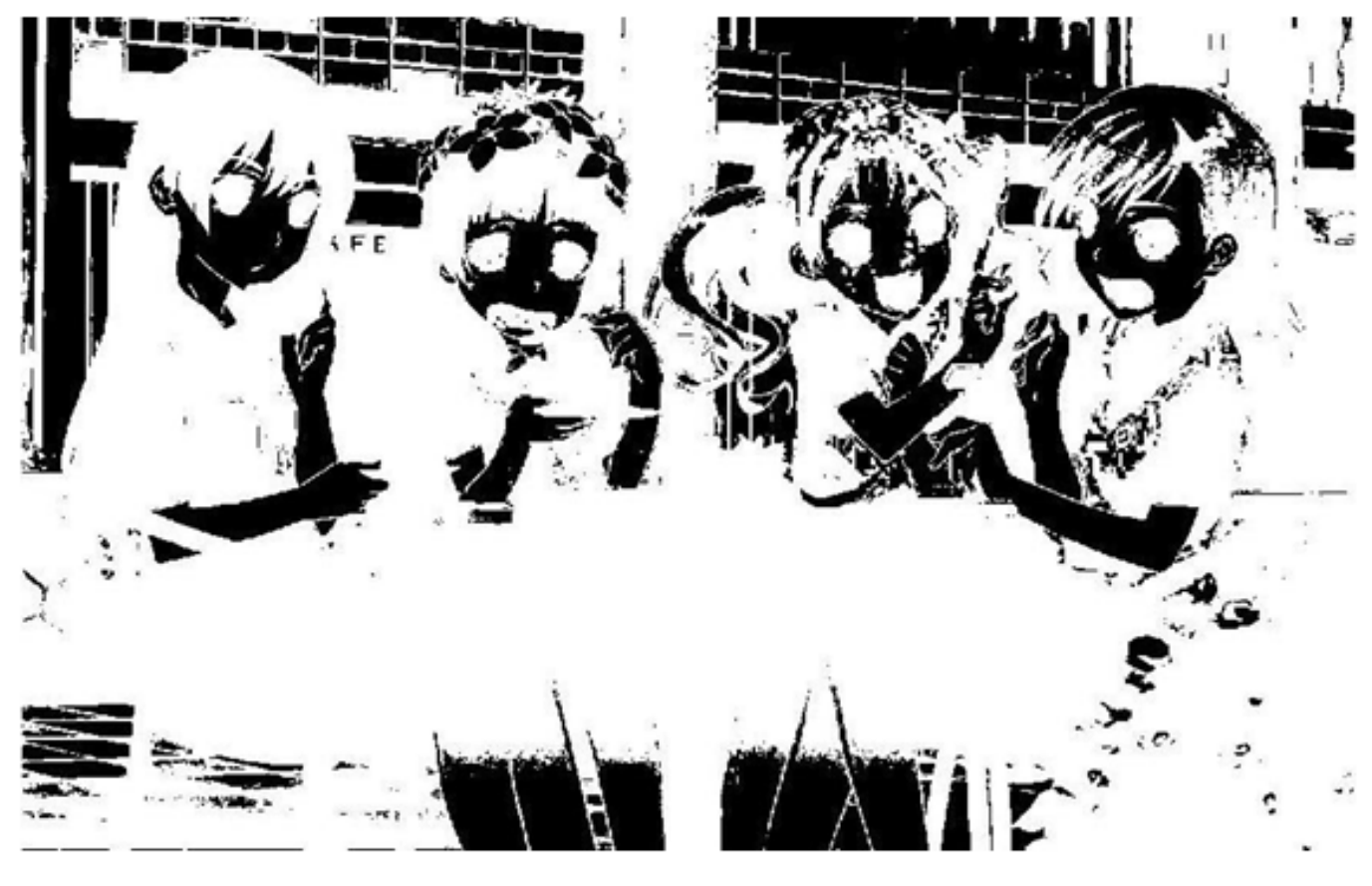

Fig. 27. Skin extracted using Takayama method

\section{Conclusions}

In this paper, we have presented three different methods to extract skin from a cartoon image. We have also showed the results of different methods in comparison with Kovac, Osman, Saleh and Takayama's methods. Our methods are based on RGB values of pixels and their comparative relations. It is evident that, only a certain range of value cannot strictly define skin in an image. We need to incorporate the shape of the character to detect the skin region. Moreover, we have also showed that, skin color can be different for different genders and races. Also, the skin color of humans and anime characters are distinctive. A method or a set of rules based on only RGB values will have limitations.

In future, we will include shape recognition of anime characters, and utilize the detected shapes along with the RGB value-based rules for skin detection. Also, we will use clustering to find similarly colored pixels so that we can set different conditions for skin for different races.

\section{REFERENCES}

[1] K. Takayama, H. Johan, and T. Nishita, "Face detection and face recognition of cartoon characters using feature extraction", Proceedings of the Institute of Image Electronics Engineers of Japan (IIEJ) Image Electronics and Visual Computing Workshop 2012, Kuching, Malaysia, November 21-24, 2012.

[2] Google Image Search, https://www.google.com/imghp

[3] J. Ruiz-del-Solar and R. Verschae, "Skin Detection using Neighborhood Information", Proceedings of the 6th International Conference on Automatic Face and Gesture Recognition (FG2004), Seoul, Korea, 2004, pp. 463-468.

[4] J. Ruiz-del-Solar and R. Verschae, "SKINDIFF-Robust and fast skin segmentation", Department of Electrical Engineering, Universidad de Chile, 2006.

[5] J. Kovac, P. Peer, and F. Solina, "Human skin colour clustering for face detection", Proceeding of EUROCON 2003, Slovenia, Ljubljana, 2003, pp. 144-148 
[6] A. Al-Shehri Saleh, "A simple and novel method for skin detection and face locating and tracking", Proceeding of Asia-Pacific Conference on Computer-Human Interaction 2004 (APCHI 2004), Springer Lecture Notes in Computer Science 3101, 2004, pp. 1-8.

[7] D. B. Swift, "Evaluating graphic image files for objectionable content", US Patent: US 7027645 B2, 2006, pp. 10.

[8] G. Osman, M.S. Hitam, and M.N. Ismail, "Enhanced skin colour classifier using RGB ratio model", International Journal on Soft Computing (IJSC), Vol.3, No.4, November 2012.

[9] N. Bourbakis, P. Kakumanu, S. Makrogiannis, R. Bryll, and S. Panchanathan, "Neural network approach for image chromatic adaptation for skin color detection", International Journal of Neural Systems, Vol. 17, No. 01, pp. 1-12, 2007.

[10] S. Shirali-Shahreza, and M.E. Mousavi, "A New Bayesian Classifier for Skin Detection", 3rd International Conference on Innovative Computing Information and Control (ICICIC'08), Dalian, China, 2008.

[11] B. Jedynak, H. Zheng, and M. Daoudi, "Skin detection using pairwise models", Image Vision Comput. 23, 13 (November 2005), pp. 1122-1130.

[12] R. Byrd and R. Balaji, "Real time 2-D face detection using color ratios and K-mean clustering", Proceedings of the 44th annual Southeast regional conference (ACM-SE 44), ACM, New York, 2006, pp. 644-648.

[13] Anime character database, http://www.animecharactersdatabase.com

[14] S. Omanovic, E. Buza, and I. Besic, "RGB ratios based skin detection", 37th International Convention on Information and Communication Technology, Electronics and Microelectronics (MIPRO), IEEE, Opatija, Croatia, 2014, pp. 1348-1353

[15] J. Canny, “A computational approach to Edge detection”, IEEE Trans. PAMI, Vol. 8, No. 6, pp. 679698, 1986.

[16] V. Vezhnevets, V. Sazonov, and A. Andreeva, "A survey on pixel-based skin color detection techniques," Graphicon, pp. 85-92, 2003.

\section{AUTHORS}

Kazi Tanvir Ahmed Siddiqui received his B.Sc. degree from North South University. He is currently working at Bangladesh University of Engineering and Technology. His research interests include Artificial Intelligence, Machine Learning and Image Processing.

Abu Wasif is currently an Assistant Professor at Bangladesh University of Engineering and Technology. His main research interests are in Artificial Intelligence and Machine Learning.

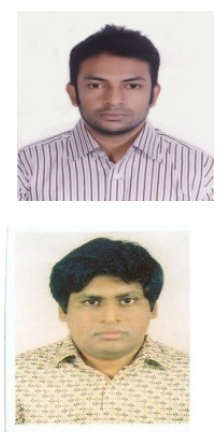

